\title{
Factors that Hinder 24-Hour Discharge After Laparoscopic Roux-en-Y Gastric Bypass: a Retrospective Analysis at a Low-Volume Center
}

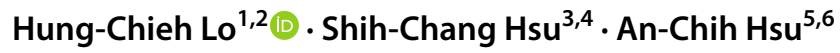

Received: 19 August 2021 / Revised: 10 November 2021 / Accepted: 14 November 2021 / Published online: 22 November 2021

(c) The Author(s), under exclusive licence to Springer Science+Business Media, LLC, part of Springer Nature 2021

\begin{abstract}
Purpose This study aimed to identify factors that hinder 24-h patient discharge after laparoscopic Roux-en-Y gastric bypass (LRYGB) in a low-volume practice.

Material and Methods Consecutive patients who fulfilled regional criteria and underwent primary LRYGB from 2018 to 2020 were retrospectively analyzed. Patients were discharged on the morning of the first postoperative day (POD1) after meeting the predefined criteria. The assessed outcome measures (POD1 vital signs, laboratory findings, pain scores and nausea/vomiting) and 30-day postoperative complications were compared between the early (stay $\leq 24 \mathrm{~h}$ ) and delayed ( $>24 \mathrm{~h}$ ) groups. Results For 107 patients who fulfilled the inclusion criteria, 48 (44.9\%) were discharged within $24 \mathrm{~h}$. There were no differences in the baseline demographics, except that the early group was more likely to have a previous abdominal operation (35.4\% vs. 16.9\%). Both groups had similar operation durations ( $89 \mathrm{~min}$ vs. $92 \mathrm{~min}$ ), but the early group had a markedly shortened length of stay (23 (24-22) h vs. 27 (47-26) h). The POD1 parameters were the same between the groups, except that the delay group had a significantly higher visual analog scale score, with fewer patient scores of 0 . Patients who were younger and female were more likely to need additional IV analgesics. No POD1 antiemesis was required throughout the study. There was no increase in the 30-day complications.

Conclusion Patient discharge at $24 \mathrm{~h}$ post-LRYGB is feasible and safe in a low-volume practice. A more comprehensive pain relief strategy may be required before generalizing this approach.
\end{abstract}

Keywords $24 \mathrm{~h} \cdot$ Bariatric $\cdot$ Low volume $\cdot$ Gastric bypass

Key points • Twenty-four-hour discharge post-LRYGB is safe for low-risk patients in a low-volume practice.

- Abdominal pain on postoperative day one hinders early patient discharge.

- Younger, female patients tend to be more susceptible to postoperative pain.

Hung-Chieh Lo

carfishcat@yahoo.com.tw

1 Division of Trauma and Emergency Surgery, Department of Surgery, Wan Fang Hospital, Taipei Medical University, Taipei 116, Taiwan

2 Department of Surgery, School of Medicine, College of Medicine, Taipei Medical University, Taipei 106, Taiwan

3 Emergency Department, Department of Emergency and Critical Medicine, Wan Fang Hospital, Taipei Medical University, Taipei 116, Taiwan

\section{Introduction}

While the world battles the COVID-19 pandemic, obesity continues to have a profound impact on public health. However, many nonemergency procedures have been postponed during the peak of the COVID-19 pandemic to accommodate the resources and capacity requirements [1]. Arguably, bariatric and metabolic surgery should be performed

4 Department of Emergency Medicine, School of Medicine, College of Medicine, Taipei Medical University, Taipei 106, Taiwan

5 Department of Anesthesiology, Wan Fang Hospital, Taipei Medical University, Taipei 116, Taiwan

6 Pain Research Center, Wan Fang Hospital, Taipei Medical University, Taipei 106, Taiwan 
for patients in need before the upcoming second pandemic wave because it has been increasingly utilized and widely accepted to be the only effective treatment for obesity and its related diseases [2]. Moreover, cumulative evidence has shown that obese populations have a higher risk of SARSCoV-2 infection and complications [3] and delayed obesity treatment during the COVID-19 pandemic worsens the preexisting comorbidities of obesity [4]. Therefore, it is vital to understand how to resume bariatric surgeries while using limited resources, reducing the risk for in-hospital infection, and maintaining the safety of the surgery.

Since their inception in 1997 [5], enhanced recovery after surgery (ERAS) protocols have successfully been shown to be a valuable modality to attenuate perioperative stress and achieve faster convalescence for various surgical disciplines [6]. With demonstrated benefits, including a shortened length of stay (LOS) and reduced overall cost [7], these protocols can be a useful and timely tool in such a situation. As evidence continues to emerge, studies have shown that it is feasible for selected patients to be discharged after $23 \mathrm{~h}$ on an outpatient basis after laparoscopic Roux-en-Y gastric bypass (LRYGB) [8-10]. However, these discharge plans after LRYGB usually occur in specialized high-volume centers and in experienced hands. In fact, looking at the Metabolic and Bariatric Surgery Accreditation and Quality Improvement Program (MBSAQIP) data registry, merely $0.9 \%$ of all LRYGBs were discharged within $24 \mathrm{~h}$ [11]. Some small retrospective studies have reported that a 1-day LOS can be accomplished for 78.1-94.8\% of patients [12]. Nevertheless, these studies either lacked a control group or did not take clinically relevant factors into consideration. While low-volume bariatric units are often less resourceful and perform no negligible bariatric procedures [13], there is still not a sufficient amount of data to support the early release of patients in these low-volume centers. With approximately 50 bariatric procedures performed yearly, surprisingly, we found that many patients met the discharge criteria earlier than expected. After full implementation of the ERAS protocols from 2017 and a year of adaptation, we routinely discharged all patients who underwent primary procedures the morning after the operation if not contraindicated. Unlike most studies focused on 30-day outcomes and the LOS [7, $11,14]$, the objective of this study was to clarify the factors that hinder 24-h patient discharge after primary LRYGB by analyzing readily accessible clinical variables and to study safety and results at the same time.

\section{Material and Methods}

A retrospective analysis was performed from a prospectively maintained database of patients who underwent bariatric surgeries from 2018 to 2020 by a single surgeon at a university affiliated hospital. Local institutional review board approval was obtained for this study, and informed consent was waived because this was a retrospective study. All procedures performed in this study were in accordance with the ethical standards in the 1964 Declaration of Helsinki and its later amendments.

Consecutive patients who met the regional criteria [15] and who underwent primary LRYGB were enrolled. Exclusion criteria included patients with BMI $>50 \mathrm{~kg} / \mathrm{m}^{2}$ who underwent procedures other than primary LRYGB. Preoperative work-up included blood tests, chest radiographs, electrocardiograms, and upper gastrointestinal endoscopy. A standard four-port LRYGB was completed in all patients with techniques including the construction of a $30-\mathrm{mL}$ vertical pouch and $100-\mathrm{cm}$ alimentary (antecolic, antegastric) and biliopancreatic limbs, the completion of linear stapling anastomoses and the closure of mesenteric defects.

As shown in Table 1, our ERAS protocol consisted of preadmission education to facilitate patient readiness for discharge and to motivate early discharge. The nasogastric tube, abdominal drain, or urinary catheters are exempted. Apart from standard anesthesia, our regimen comprised the monitoring of optimal muscle tension during surgery, the depth of anesthesia, the attention to the pre-emptive antiemesis protocol, and the use of a multimodal analgesic regimen based upon reported evidence at the time [16]. Patients were encouraged to proceed with early ambulation with no thromboprophylaxis postoperatively, and a liquid diet commenced on the first postoperative day (POD1). A goal to achieve at least $1500-\mathrm{ml}$ total fluid per day was given, which in turn represents approximately 100-ml/hour, considering approximately $16 \mathrm{~h}$ of daytime activity. The postoperative diet regimen consisted of four stages of advancement, with a clear liquid diet serving as the first stage and then slowly advancing after discharge until accustomed to a pureed diet across a 16-day time frame. Discharge was prepared in advance on the day of surgery on a regular basis and granted on the morning of the POD1 based upon reach of diet milestone and if the patient had no fever and had a stable hemodynamic status with tolerable pain and if the laboratory results were within the acceptable range. As the ideal of this research is to conduct such practice under a low-volume practice, rotation staff is not often available. The attending physician personally confirms the suitability of early release and collaborates to handle discharge. For any patients who were subjectively unwell or had concerning characteristics, we initiated a timely work-up. For these patients, a same-day discharge was still allowed after a negative secondary survey and with improved clinical status. Patients were stratified into early ( $\leq 24$ hours) or delayed ( $>24$ hours) groups based on the postoperative LOS, which was measured in hours and was defined as the time from the end of the procedure to the exact time the patient left 
Table 1. ERAS protocol

\begin{tabular}{|c|c|c|}
\hline Phase of care & ERAS goal (content) & Route (dosage) \\
\hline Preadmission & \multicolumn{2}{|l|}{ Instructions to provide clear expectations and goals } \\
\hline Intra-operation & \multicolumn{2}{|l|}{ No nasogastric tube } \\
\hline & \multicolumn{2}{|l|}{ No Foley catheter } \\
\hline & \multicolumn{2}{|l|}{ No abdominal drain } \\
\hline \multicolumn{3}{|l|}{ Anesthesia } \\
\hline Deep neuromuscular blockage & Optimal muscle tension (train of four monitor) & IV Sugammadex (2-4 mg/kg) \\
\hline Depth of anesthesia & \multicolumn{2}{|l|}{ Bispectral index } \\
\hline Pre-emptive anti-emesis protocol & \multicolumn{2}{|l|}{ Goal directed fluid replacement (total < $1.5 \mathrm{~L}$ ) } \\
\hline Induction & Dexamethasone & IV $10 \mathrm{mg}$ \\
\hline Before the end of surgery & Droperidol & IV $0.625-2.5 \mathrm{mg}$ \\
\hline \multirow[t]{2}{*}{ Postoperative } & Metoclopramide & IV $10 \mathrm{mg} \mathrm{q} 8 \mathrm{~h}$ \\
\hline & Granisetron & IV $3 \mathrm{mg}$ and QD on demand \\
\hline Multimodal analgesic regimen & \multicolumn{2}{|l|}{ Total elimination of opioid use } \\
\hline \multirow[t]{3}{*}{ Preoperative } & Clonidine & po $75 \mathrm{mcg}$ \\
\hline & Pregabalin & po $75 \mathrm{mg}$ \\
\hline & Acetaminophen & IV $1000 \mathrm{mg}$ \\
\hline \multirow[t]{2}{*}{ Induction } & Propofol & IV $1 \% 1-2.5 \mathrm{mg} / \mathrm{kg}$ \\
\hline & Fentanyl & IV $1-2 \mathrm{mcg} / \mathrm{kg}$ \\
\hline \multirow[t]{3}{*}{ Intraoperative } & Transverses abdominis plane block & Bupivacaine, $0.5 \%$, total $40 \mathrm{ml}$ \\
\hline & Port site local injection & \\
\hline & Intraperitoneal spray & One-time spray after pneumoperitoneum \\
\hline \multirow[t]{2}{*}{ At the end of procedure } & Fentanyl & IV $50 \mathrm{mcg}$ on demand \\
\hline & Ketorolac & IV $30 \mathrm{gm}$ on demand \\
\hline \multirow[t]{2}{*}{ Postoperative } & Parecoxib & IV $40 \mathrm{gm}$ and QD on demand \\
\hline & Acetaminophen & IV $1000 \mathrm{mg}$ q6 h on demand \\
\hline \multicolumn{3}{|l|}{ Patient care } \\
\hline Postoperative & \multicolumn{2}{|l|}{ Sip water, rigorous early ambulation } \\
\hline POD1 & \multicolumn{2}{|c|}{ Clear liquids, routine lab survey, handout discharge instructions, oral acetaminophen } \\
\hline
\end{tabular}

ERAS enhanced recovery after surgery, $I V$ intravenous, $P O D 1$ postoperative day one

the hospital. Demographics assessed included the estimated glomerular filtration rate (eGFR), stage of chronic kidney disease (CKD), and baseline oxygen saturation $\left(\mathrm{O}_{2}\right)$ as surrogates of major organ function and American Society of Anesthesiologists (ASA) physical status classification. The primary outcomes evaluated included POD1 vital signs and laboratory survey results. Having been demonstrated to be a reliable and valid measure for acute pain and limited by its retrospective design, the patient-reported intensity of abdominal pain is rated according to the visual analog scale (VAS) [17] with increments of severity ranging from 0 to 10 , and the use of analgesic or anti-emetic medications is collected. The 30-day complications served as the secondary outcome measures. Patients were followed postoperatively at 1 week and 1, 3, 6, and 12 months postoperatively and then annually thereafter.

Statistical analysis was performed using R 4.1.0 software (R Foundation for Statistical Computing, Vienna, Austria). The normality of the data was assessed by the Shapiro-Wilk test. Continuous variables were expressed as the means and standard deviation or medians and the interquartile range when appropriate and were compared by Student's $t$ test or the Mann-Whitney U-test. Categorical variables were expressed as counts or percentages and analyzed with the chi-squared test or Fisher's direct exact test. Statistical tests were two-sided, and a value of $p<0.05$ was considered statistically significant.

\section{Results}

From 2018 to 2020, a total of 168 patients underwent bariatric surgery at our hospital. Among 107 who fulfilled the inclusion criteria, 48 (44.9\%) were discharged within $24 \mathrm{~h}$, and the other 59 (55.1\%) stayed more than $24 \mathrm{~h}$.

The demographic features and clinical characteristics are outlined in Table 2. No significant differences were found between the groups with respect to age, female sex, body 
Table 2. Clinical characteristics of the patients

\begin{tabular}{|c|c|c|c|}
\hline Variables & $\begin{array}{l}\text { Early group } \\
(N=48)\end{array}$ & $\begin{array}{l}\text { Delayed group } \\
(N=59)\end{array}$ & $P$ value \\
\hline Age (years), median (IQR) & $38(42.3-31.0)$ & $36.0(42.5-30.0)$ & 0.660 \\
\hline Female, n $(\%)$ & $31(64.6)$ & $36(61.0)$ & 0.858 \\
\hline BMI $\left(\mathrm{kg} / \mathrm{m}^{2}\right)$, median $(\mathrm{IQR})$ & $36.6(38.8-34.9)$ & $37.3(40.0-34.9)$ & 0.535 \\
\hline Prior abdominal operation, n (\%) & $17(35.4)$ & $10(16.9)$ & $0.049 *$ \\
\hline Visceral & 4 & 2 & 0.820 \\
\hline Gynecological & 11 & 8 & \\
\hline Others & 2 & & \\
\hline \multicolumn{4}{|l|}{ Comorbidity, n (\%) } \\
\hline Diabetes mellitus & $14(29.2)$ & $18(30.5)$ & 1.00 \\
\hline Insulin & 1 & 4 & 0.354 \\
\hline OHA & 13 & 14 & \\
\hline Hypertension & $18(37.5)$ & $24(40.7)$ & 0.892 \\
\hline Dyslipidemia & $34(70.8)$ & $35(59.3)$ & 0.300 \\
\hline GERD & $22(45.8)$ & $30(50.8)$ & 0.747 \\
\hline eGFR $\left(\mathrm{mL} / \mathrm{min} / 1.73 \mathrm{~m}^{2}\right)$, mean $(\mathrm{SD})$ & $121.0(27.0)$ & $113.9(23.5)$ & 0.155 \\
\hline CKD stage 1 & 19 & 26 & 0.754 \\
\hline CKD stage 2 & 6 & 9 & \\
\hline CKD stage 3 & 0 & 1 & \\
\hline $\mathrm{O}_{2}$ sat $(\%)$, median (IQR) & $96.0(98.0-96.0)$ & $97.0(98.5-96.0)$ & 0.394 \\
\hline \multicolumn{4}{|l|}{ ASA, n (\%) } \\
\hline II & $39(81.3)$ & $47(79.7)$ & 1.00 \\
\hline III & $9(18.7)$ & $12(20.3)$ & \\
\hline
\end{tabular}

$A S A$ American Society of Anesthesiologists Classification, $B M I$ body mass index, $C K D$ chronic kidney disease, $e G F R$ estimated glomerular filtration rate, GERD gastroesophageal reflux disease, $O H A$ oral hypoglycemic agent, $\mathrm{O}_{2}$ sat oxygen saturation

$* P<0.05$

mass index (BMI), or incidence of comorbidities, except that the early group had significantly more prior abdominal operations (17 (35.4\%) vs. $10(16.9 \%) ; p=0.049)$, which were mostly gynecological procedures due to exclusively benign lesions. In our study, no patients required changes in their treatment plan throughout the study. There were also no differences in the eGFR, stage of CKD, $\mathrm{O}_{2}$ saturation, or ASA between the groups.

The surgical results and POD1 parameters are depicted in Table 3. There was an equivalent median operation time ( 89 min vs. $92 \min ; p=0.636$ ) between the groups and the LOS was markedly shortened in the early group (23 (24-22) h vs. 27 (47-26) h; $p<0.001)$. There was no difference in vital signs or laboratory data, including white blood cell (WBC) count, percentages of neutrophils, $\mathrm{C}$-reactive protein (CRP), hemoglobin ( $\mathrm{Hb})$ decline, and $\mathrm{Hb}$ value at baseline or on POD1 between the groups. The early group had a significantly lower POD1 pain scale (1 $(1-0)$ vs. $1(2-1) ; p=0.001)$ and had more patients with a pain scale of $0(31.3 \%$ vs. $8.5 \%$; $p=0.005)$. As both pain relievers (e.g., parecoxib and acetaminophen) were given on demand after surgery, there was a trend in the early group for a less frequent request for these analgesics $(2.1 \%$ vs. $11.9 \%$; $p=0.071$ ) on the morning of POD1. Apart from one patient in each group who required a single dose of morphine, each group had another patient; one in the early group received ketorolac, and the other in the delay group received tramadol to control breakthrough pain at the night of the operation. None of the other patients received any other pain relievers outside of our protocol or had a requirement for antiemetic medications. The incidence of 30-day ER visits, readmissions, and overall complications ( $1(2.1 \%)$ vs. $3(5.1 \%) ; p=0.626)$ were the same between the groups. Complications included one patient with hematochezia in the early group who required readmission via the ER. In the delay group, two patients were readmitted via the ER for self-limiting hematemesis. A third patient experienced transient melena at POD7. There was no anastomotic leakage, open conversion or mortality reported in any of the patients. At the 1-year follow-up, the percentage of excessive weight loss and the percentage of total weight loss in either group were $77.7 \%$ and $27.9 \%$ vs. $85.6 \%$ and $31.8 \%$, respectively. 
Table 3. Surgical perspectives, outcomes and POD1 parameters

\begin{tabular}{llll}
\hline Variables & Early group & Delayed group & $P$ value \\
\hline Operative duration (min), median (IQR) & $89.0(97.5-82.8)$ & $92.0(103.0-81.5)$ & 0.636 \\
LOS (hours), median (IQR) & $23(24-22)$ & $27(47-26)$ & $<0.001^{*}$ \\
POD1 parameters & & & \\
BT $\left({ }^{\circ} \mathrm{C}\right)$, median (IQR) & $36.6(36.8-36.4)$ & $36.6(36.8-36.4)$ & 0.879 \\
PR (bpm), mean (SD) & $73.3(11.1)$ & $71.5(11.5)$ & 0.406 \\
RR (BPM), median (IQR) & $16(18-16)$ & $17(18-16)$ & 0.724 \\
SBP (mmHg), mean (SD) & $124.8(14.6)$ & $124.8(14.9)$ & 0.993 \\
WBC (103/ul), median (IQR) & $12.62(14.15-10.73)$ & $12.54(14.19-10.84)$ & 0.873 \\
$>14,000, \mathrm{n}(\%)$ & $14(29.2 \%)$ & $17(28.8 \%)$ & 1.00 \\
Neutrophil \%, median (IQR) & $76.6(79.9-72.9)$ & $77.5(79.3-73.3)$ & 0.843 \\
$>85 \%, \mathrm{n}(\%)$ & $3(6.3 \%)$ & $1(1.7 \%)$ & 0.323 \\
Hb baseline (g/dl), median (IQR) & $14.5(15.6-13.9)$ & $14.5(15.8-13.9)$ & 0.633 \\
Hb POD1, median (IQR) & $13.0(13.9-12.4)$ & $13.0(13.9-12.3)$ & 0.702 \\
Hb decline $>2, \mathrm{n}(\%)$ & $9(18.8)$ & $13(22.0)$ & 0.859 \\
CRP (mg/dl), median (IQR) & $1.5(2.2-1.3)$ & $1.5(2.4-1.1)$ & 0.746 \\
CRP >6, n (\%) & $3(6.3)$ & $2(3.4)$ & 0.655 \\
VAS, median (IQR) & $1(1-0)$ & $1(2-1)$ & $0.001^{*}$ \\
VAS 0, n (\%) & $15(31.3)$ & $5(8.5)$ & $0.005^{*}$ \\
Analgesics requirement, n (\%) & $1(2.1)$ & $7(11.9)$ & 0.071 \\
Anti-emesis, n (\%) & 0 & 0 & 1.00 \\
30-day ER visits, n (\%) & $1(2.1)$ & $4(6.8)$ & 0.376 \\
$30-$ day readmission, n (\%) & $1(2.1)$ & $2(3.4)$ & 1.00 \\
30-day complications, n (\%) & $1(2.1)$ & $3(5.1)$ & 0.626 \\
\hline
\end{tabular}

$B P M$ breaths per minute, $B T$ body temperature, $C R P$ C-reactive protein, $E R$ emergency room, $H b$ hemoglobin, $L O S$ length of stay, $P O D 1$ postoperative day one, $P R$ pulse rate, $R R$ respiratory rate, $S B P$ systolic blood pressure, VAS Visual Analog Scale, WBC white blood cell

$* P<0.05$

\section{Discussion}

With a shortened hospital stay and no increase in complications, our data support the feasibility and safety of 24-h discharge after LRYGB in a low-volume setting. Postoperative abdominal pain appears to be the only causative factor in our study that affects early discharge. In this regard, a collaborative multidisciplinary approach for more satisfactory pain relief should help more suitable patients be discharged early.

LRYGB is considered the gold-standard bariatric procedure and is performed only second to sleeve gastrectomy. LRYGB generally has an LOS of 2 days [8] and has a higher risk profile than sleeve gastrectomy [18]. Systemic reviews suggest that it is feasible to discharge patients within $23 \mathrm{~h}$ after primary LRYGB [7, 12]. However, controversies continue regarding its drawbacks. For instance, Morton et al. [8] discovered that a postoperative LOS of $\leq 1$ day was associated with a significantly increased risk of 30-day mortality. Inaba et al. [14] also found that there was a significantly higher risk-adjusted overall morbidity and readmission for same-day discharge using the MBSAQIP database. In contrast, other studies have shown no increase in 30-day morbidities under this approach [11, 19]. Importantly, the nature of abdominal symptoms can be vague and insidious because of obesity. Since independent vital signs such as a heart rate of $>120 / \mathrm{min}$, respiratory distress (respiratory rate $>22 / \mathrm{min}$ ) [20] or a combination of these are frequently referred to as essential indicators in identifying complications early, we paid extra attention to these parameters and conducted this approach cautiously. Corresponding to the overall low complications, none of our patients experienced fever, dyspnea, tachycardia, or unstable blood pressure on POD1; additionally, there was no difference in these factors between the groups. Furthermore, despite the major disadvantage of the lack of specificity [21] and the optimal detection time and standard value not being conclusive [22], we routinely performed blood sampling on POD1 and interpreted the results based on strict reported standards, including a WBC count $>14 \times 10^{3} / \mu \mathrm{L}$ [21], percentage of neutrophils $>85 \%, \mathrm{CRP}>6 \mathrm{mg} / \mathrm{dl}$ [23] and serial $\mathrm{Hb}$ decline $>2 \mathrm{~g} / \mathrm{dL}$ [23]. These factors were used as complementary tools to help screen patients who could have complications. Further analysis revealed that none of these factors reached statistical significance or correlated directly with the 
development of complications. However, the current study may be underpowered to detect significance given the low patient number and the low incidence of complications in this study. In referring to previous large studies with reported readmission rates ranging between 1.7 and $5.6 \%$, reoperation between 1.02 and $2.49 \%$, overall complication rates between 1.9 and $3.76 \%$, and mortality rates up to $0.94 \%[9-11,14$, 19], our results fall within these ranges and therefore meet the safety standards.

Importantly, as prerequisites for successful and safe early patient discharge, there are currently no established patient selection or exclusion criteria. While multivariate analysis proposed that surgeon experience $<50$ cases, patient age $>$ 56 , BMI $>60$, patient weight $>400 \mathrm{lbs}$, and patient comorbidities $>4$ were unfavorable factors for early discharge [10], there was no difference in the patients' demographics that reflected these conditions between the groups. In other words, we did not select lower-risk patients to achieve the goal of early discharge. Notably, the patients in our study had a lower median BMI in both groups (36.6 and $37.3 \mathrm{~kg} /$ $\mathrm{m}^{2}$, respectively) than previous reports that described patient BMIs ranging from 41.2 to $49.3 \mathrm{~kg} / \mathrm{m}^{2}$ [7]. Part because a body mass index (BMI) $>50 \mathrm{~kg} / \mathrm{m}^{2}$ was not considered suitable for LRYGB in our practice because gastric bypass is generally less effective in these patients [24], and these patients generally have a higher rate of complications than patients with a lower BMI [25]. Since only $19 \%$ of patients in the early group (BMI range, $32.8-45.9 \mathrm{~kg} / \mathrm{m}^{2}$ ) and $25 \%$ of patients in the delay group (BMI range, $32.5-48.1 \mathrm{~kg} /$ $\mathrm{m} 2$ ) have BMI $>40 \mathrm{~kg} / \mathrm{m}^{2}$ and because most of our patients belonged to ASA class II, our patient groups appear to have had a lower risk, and such practices are in line with the aforementioned recommendations [7, 10]. Conversely, after taking into account the reported high discharge rate, for instance, McCarty et al. [10] proposed that $84 \%$ of 2000 LRYGB patients can be successfully discharged within 23 h, and Hahl et al. [26] reported a high POD1 discharge rate of up to $83 \%$ in a 318 patient series; our study had inferior results since only $44.9 \%$ of our patients were discharged within $24 \mathrm{~h}$. Part of the reason for our data may be because we reported the LOS by the hour, and we are less experienced. Intergroup analysis revealed that postoperative pain serves as the only factor that hinders early discharge despite both groups having no underlying differences in the patient demographics, using the same multimodal analgesics regimen, having a generally low pain scale, and specifically, only $11.9 \%$ of patients in the delayed group requested extra POD 1 IV analgesics. Moreover, none of these patients experienced 30-day complications, and none of our patients required additional imaging studies or interventions as a cause of lengthier stays. In addition, we divided the cohort into tertiles and compared the tertile with the shortest LOS (median LOS 23 hours) with the highest LOS (median LOS
46 hours) (Table 4). The demographics were no differing between groups. In the comparison of POD1 parameters, similarly, we found that the significant factors were a higher VAS score with a lower patient score of 0 in the 3rd tertile group than in the 1st tertile group. The disadvantage is that we did not distinguish the nature of abdominal pain, and the clinical treatment of various pain types was roughly the same during the study period. Intragroup analysis revealed that those patients who required POD1 analgesics had a higher pain scale $(2(3-2)$ vs. $1(2-1) ; p<0.001)$ and tended to be younger (33.0 years vs. 37.5 years; $p=0.124$ ) and female ( $71.4 \%$ vs. $59.6 \% ; p=0.850)$, but the sample size was too small to reach statistical significance. Interestingly, younger patients ( $<40$ years) and female patients were formerly recognized as independent factors associated with postoperative pain [27]. Because our analgesic regimen is solely based on one long-acting and another short-acting drug that complements each other, a more liberal use of perioperative nonopioid adjuvants at regular intervals independent of the severity of pain may offer improvements in patient satisfaction levels and further enhance early discharge [16]. There are likely many other uncontrollable factors that may change the willingness to discharge a patient, such as insurance reimbursement, distance [28] and patient choice [7], but the conditions were the same between the groups. Since the clinical team may have conflicting responsibilities on different days, a further analysis was performed to determine whether weekdays or weekends affect the timeliness of discharge. Paradoxically, more patients (17/48) in the early group were discharged on weekends than in the delay group (10/59) $(p=0.049)$. Overall, there was a marginally shorter LOS for those discharged on weekends $(n=27)$ than for those discharge on weekdays $(n=80)(24$ (25.5-23) hours vs. 26 (28-23) hours; $p=0.08$ ). Moreover, regarding the time of day the case concluded, a significantly more patients in early group (19/48) conclude their index operation after noon than delay group $(11 / 59)(p<0.001)$. In other words, these factors that seem not conducive to discharge do not deter early discharge in the current research. On the other hand, while postoperative nausea/vomiting and dehydration are also common complications that discourage early discharge [29], remarkably, no patient in our study required POD1 anti-emetic drugs or had problems in oral hydration despite objective nausea scores not being evaluated in our study. Part of our success can be attributed to the full adherence to an established protocol consisting of a pre-emptive anti-emetic protocol, limited intravenous fluid administration, and the near total elimination of opioid use. Affected by the way the data presented, it appears the research discussion revolve around only $4 \mathrm{~h}$ difference in LOS. In fact, nearly half of the patients (24/59) in the delay group were discharged on POD2. As a community-based hospital with limited beds (total=284), we believe such advances have a 
Table 4. Surgical perspectives, outcomes, and POD1 parameters between tertiles

\begin{tabular}{llll}
\hline Variables & 1 st tertile & 3 rd tertile & $P$ value \\
\hline Operative duration (min), median (IQR) & $89.5(99.0-82.0)$ & $92.0(100.3-87.0)$ & 0.573 \\
LOS (hours), median (IQR) & $23(23-21)$ & $46(48-28)$ & $<0.001^{*}$ \\
POD1 parameters & & & \\
BT $\left({ }^{\circ} \mathrm{C}\right)$, median (IQR) & $36.6(36.7-36.4)$ & $36.6(36.8-36.4)$ & 0.843 \\
PR (bpm), mean (SD) & $73.0(9.4)$ & $71.3(11.6)$ & 0.506 \\
RR (BPM), median (IQR) & $16(17-16)$ & $17(18-16)$ & 0.365 \\
SBP (mmHg), mean (SD) & $125.5(14.1)$ & $122.3(14.7)$ & 0.375 \\
WBC (103/ul), median (IQR) & $12.70(14.26-10.82)$ & $12.83(14.23-11.17)$ & 0.942 \\
$>14,000, \mathrm{n}(\%)$ & $5(13.9)$ & $0(0)$ & 0.054 \\
Neutrophil \%, median (IQR) & $77.5(80.6-73.2)$ & $77.9(79.7-73.3)$ & 0.991 \\
$>85 \%, \mathrm{n}(\%)$ & $3(8.3)$ & $1(2.8)$ & 0.614 \\
Hb baseline (g/dl), median (IQR) & $14.6(15.4-14.0)$ & $14.7(16.1-13.9)$ & 0.338 \\
Hb POD1, median (IQR) & $13.1(13.8-12.5)$ & $13.2(14.1-12.5)$ & 0.744 \\
Hb decline $>2, \mathrm{n}(\%)$ & $4(11.1)$ & $10(27.8)$ & 0.137 \\
CRP (mg/dl), median (IQR) & $1.6(2.3-1.3)$ & $1.5(2.4-1.1)$ & 0.685 \\
CRP >6, n (\%) & $3(8.3)$ & $1(2.8)$ & 0.614 \\
VAS, median (IQR) & $1(1-0)$ & $2(2-1)$ & $<0.001^{*}$ \\
VAS 0, n (\%) & $11(30.6)$ & $1(2.8)$ & $0.004^{*}$ \\
Analgesics requirement, n (\%) & $1(2.8)$ & $5(13.9)$ & 0.199 \\
Anti-emesis, n (\%) & 0 & 0 & 1.00 \\
30-day ER visits, n (\%) & $1(2.8)$ & $3(8.3)$ & 0.614 \\
$30-$ day readmission, n (\%) & $1(2.8)$ & $1(2.8)$ & 1.00 \\
$30-$ day complications, n (\%) & $1(2.8)$ & $1(2.8)$ & 1.00 \\
\hline
\end{tabular}

$B P M$ breaths per minute, $B T$ body temperature, $C R P$ C-reactive protein, $E R$ emergency room, $H b$ hemoglobin, $L O S$ length of stay, $P O D 1$ postoperative day one, $P R$ pulse rate, $R R$ respiratory rate, $S B P$ systolic blood pressure, VAS Visual Analog Scale, WBC white blood cell

$* P<0.05$ significant impact on the flow of the ward and facilitate us to use limited resources more efficiently.

\section{Limitations}

Inherent to its retrospective and nonrandomized nature, selection bias cannot be completely eliminated. It is also possible that the differing nature of vague abdominal symptoms and subjective evaluation of pain scales based on different staff members are prone to inconsistent results. The lower BMI and relatively low-risk subgroup further limit the generalizability of our study. Although not ideally designed, the strengths of this study reside in the full adherence to a standard protocol, the surgery being performed by a single surgeon, and the study being conducted across a short time span. Therefore, the introduction of major flaws in this study from heterogeneous practices or from advances in surgical techniques and comprehensive care over time can largely be avoided.

In conclusion, our study favors the safety and feasibility of patient discharge within $24 \mathrm{~h}$ for low-risk patients in a low-volume setting. A more comprehensive protocol, such as administering additional nonopioid medications irrespective of the pain score, may further generalize this approach. Studies with more robust data and a longer follow-up should be performed in the future to support this discharge practice.

Authors' Contributions Author 1 designed the study, performed the surgical procedures, followed the patients, and participated in the data analysis and in the writing of the manuscript. Author 2 contributed to the data collection and analysis. Author 3 contributed to the data collection and analysis. All authors have read and approved the final version to be published.

Funding The study was not sponsored and funded by any funding.

\section{Declarations}

Ethical Approval All procedures performed in studies involving human participants were in accordance with the ethical standards of institutional and/or national research committees and with the 1964 Declaration of Helsinki and its later amendments or comparable ethical standards. 
Informed Consent Informed consent was waived because no identifiable data of the patients were disclosed.

Conflict of Interest Dr. Hung-Chieh Lo, Dr. Shih-Chang Hsu, and Dr. An-Chih Hsu declare that they have no conflicts of interest.

\section{References}

1. Yang W, Wang C, Shikora S, Kow L. Recommendations for metabolic and bariatric surgery during the covid-19 pandemic from IFSO. Obes Surg. 2020;30(6):2071-3.

2. Buchwald H, Avidor Y, Braunwald E, Jensen MD, Pories W, Fahrbach K, Schoelles K. Bariatric surgery: a systematic review and meta-analysis. JAMA. 2004;292(14):1724-37.

3. Lighter J, Phillips M, Hochman S, Sterling S, Johnson D, Francois $\mathrm{F}$, Stachel A. Obesity in patients younger than 60 years is a risk factor for covid-19 hospital admission. Clin Infect Dis. 2020;71(15):896-7.

4. Rubino F, Cohen RV, Mingrone G, le Roux CW, Mechanick JI, Arterburn DE, Vidal J, Alberti G, Amiel SA, Batterham RL, Bornstein S, Chamseddine G, Del Prato S, Dixon JB, Eckel RH, Hopkins D, McGowan BM, Pan A, Patel A, et al. Bariatric and metabolic surgery during and after the COVID-19 pandemic: DSS recommendations for management of surgical candidates and postoperative patients and prioritisation of access to surgery. Lancet Diabetes Endocrinol. 2020;8(7):640-8.

5. Kehlet H. Multimodal approach to control postoperative pathophysiology and rehabilitation. Br J Anaesth. 1997;78(5):606-17.

6. Malczak P, Pisarska M, Piotr M, Wysocki M, Budzynski A, Pedziwiatr M. Enhanced recovery after bariatric surgery: systematic review and meta-analysis. Obes Surg. 2017;27(1):226-35.

7. Elliott JA, Patel VM, Kirresh A, Ashrafian H, Le Roux CW, Olbers T, Athanasiou T, Zacharakis E. Fast-track laparoscopic bariatric surgery: a systematic review. Updates Surg. 2013;65(2):85-94.

8. Morton JM, Winegar D, Blackstone R, Wolfe B. Is ambulatory laparoscopic Roux-en-Y gastric bypass associated with higher adverse events? Ann Surg. 2014;259(2):286-92.

9. Raftopoulos I, Giannakou A, Davidson E. Prospective 30-day outcome evaluation of a fast-track protocol for 23-hour ambulatory primary and revisional laparoscopic Roux-en-Y gastric bypass in 820 consecutive unselected patients. J Am Coll Surg. 2016;222(6):1189-200.

10. McCarty TM, Arnold DT, Lamont JP, Fisher TL, Kuhn JA. Optimizing outcomes in bariatric surgery: outpatient laparoscopic gastric bypass. Ann Surg. 2005;242(4):494-8. discussion 498-501

11. Barbat S, Thompson KJ, McKillop IH, Kuwada TS, Gersin K, Nimeri A. Ambulatory bariatric surgery: does it really lead to higher rates of adverse events? Surg Obes Relat Dis. 2020;16(11):1713-20.

12. Thomas H, Agrawal S. Systematic review of 23-hour (outpatient) stay laparoscopic gastric bypass surgery. J Laparoendosc Adv Surg Tech A. 2011;21(8):677-81.

13. Jafari MD, Jafari F, Young MT, Smith BR, Phalen MJ, Nguyen NT. Volume and outcome relationship in bariatric surgery in the laparoscopic era. Surg Endosc. 2013;27(12):4539-46.

14. Inaba CS, Koh CY, Sujatha-Bhaskar S, Zhang L, Nguyen NT. Same-day discharge after laparoscopic roux-en-y gastric bypass: an analysis of the metabolic and bariatric surgery accreditation and quality improvement program database. J Am Coll Surg. 2018;226(5):868-73.
15. Kasama K, Mui W, Lee WJ, Lakdawala M, Naitoh T, Seki Y, Sasaki A, Wakabayashi G, Sasaki I, Kawamura I, Kow L, Frydenberg H, Chen A, Narwaria M, Chowbey P. IFSO-APC consensus statements 2011. Obes Surg. 2012;22(5):677-84.

16. Budiansky AS, Margarson MP, Eipe N. Acute pain management in morbid obesity - an evidence based clinical update. Surg Obes Relat Dis. 2017;13(3):523-32.

17. Bijur PE, Silver W, Gallagher EJ. Reliability of the visual analog scale for measurement of acute pain. Acad Emerg Med. 2001;8(12):1153-7.

18. Kumar SB, Hamilton BC, Wood SG, Rogers SJ, Carter JT, Lin MY. Is laparoscopic sleeve gastrectomy safer than laparoscopic gastric bypass? A comparison of 30-day complications using the MBSAQIP data registry. Surg Obes Relat Dis. 2018;14(3):264-9.

19. Leepalao MC, Arredondo D, Speights F, Duncan TD. Same-day discharge on laparoscopic Roux-en-Y gastric bypass patients: an outcomes review. Surg Endosc. 2020;34(8):3614-7.

20. Hamilton EC, Sims TL, Hamilton TT, Mullican MA, Jones DB, Provost DA. Clinical predictors of leak after laparoscopic Roux-en-Y gastric bypass for morbid obesity. Surg Endosc. 2003;17(5):679-84.

21. Wysocki M, Malczak P, Wierdak M, Waledziak M, Hady HR, Diemieszczyk I, Proczko-Stepaniak M, Szymanski M, Dowgiallo-Wnukiewicz N, Szeliga J, Pedziwiatr M, Major P. Utility of inflammatory markers in detection of perioperative morbidity after laparoscopic sleeve gastrectomy, laparoscopic Roux-en-Y gastric bypass, and one-anastomosis gastric bypass-multicenter study. Obes Surg. 2020;30(8):2971-9.

22. Bona D, Micheletto G, Bonitta G, Panizzo V, Cavalli M, Rausa E, Cirri S, Aiolfi A. Does C-reactive protein have a predictive role in the early diagnosis of postoperative complications after bariatric surgery? Systematic review and bayesian meta-analysis. Obes Surg. 2019;29(11):3448-56.

23. Sroka G, Milevski D, Shteinberg D, Mady H, Matter I. Minimizing hemorrhagic complications in laparoscopic sleeve gastrectomy--a randomized controlled trial. Obes Surg. 2015;25(9):1577-83.

24. Magro DO, Geloneze B, Delfini R, Pareja BC, Callejas F, Pareja JC. Long-term weight regain after gastric bypass: a 5-year prospective study. Obes Surg. 2008;18(6):648-51.

25. Schwartz ML, Drew RL, Chazin-Caldie M. Factors determining conversion from laparoscopic to open Roux-en-Y gastric bypass. Obes Surg. 2004;14(9):1193-7.

26. Hahl T, Peromaa-Haavisto P, Tarkiainen P, Knutar O, Victorzon M. Outcome of laparoscopic gastric bypass (LRYGB) with a program for enhanced recovery after surgery (ERAS). Obes Surg. 2016;26(3):505-11.

27. Hartwig M, Allvin R, Bäckström R, Stenberg E. Factors associated with increased experience of postoperative pain after laparoscopic gastric bypass surgery. Obes Surg. 2017;27(7):1854-8.

28. Moraes IDS, Madalosso CA, Palma LA, Fornari ACDS, Dourado MDS, Scherer T, Gurski RR, Fornari F. Hospital discharge in the day following open Roux-en-Y gastric bypass: is it feasible and safe? Obes Surg. 2009;19(3):281-6.

29. Aman MW, Stem M, Schweitzer MA, Magnuson TH, Lidor AO. Early hospital readmission after bariatric surgery. Surg Endosc. 2016;30(6):2231-8.

Publisher's Note Springer Nature remains neutral with regard to jurisdictional claims in published maps and institutional affiliations. 